\title{
Perfil sociodemográfico e clínico de pacientes com câncer internados em uma Unidade de Terapia Intensiva adulto
}

\author{
Sociodemographic and clinical profile of cancer patients admitted to the adult Intensive \\ Care Unit
}

Perfil Sociodemográfico y clínico de los pacientes con cancer internados en la Unidad de Cuidados Intensivo de adultos

Laura Beatriz Sousa de Jesus Martelletti ${ }^{1 *}$, Luísa Resende Martinello ${ }^{1}$, Larissa Cristine Guimarães dos Santos ${ }^{1}$, Ana Aurora Reis Campos Neves Ferrão ${ }^{1}$, Juliana Mendes Pereira ${ }^{1}$, Carla Targino Bruno dos Santos ${ }^{1}$, Keila Cristianne Trindade da Cruz ${ }^{1}$.

\section{RESUMO}

Objetivos: Identificar o perfil sociodemográfico e clínico dos pacientes oncológicos admitidos em uma Unidade de Terapia Intensiva adulto, no ano de 2015. Método: Trata-se de um estudo descritivo, retrospectivo e transversal, com abordagem quantitativa, baseado na análise de prontuários. O estudo foi desenvolvido em um hospital universitário do Distrito Federal, no Serviço de Arquivo Médico. A população do estudo foi composta por todos os prontuários de pacientes oncológicos admitidos na Unidade de Terapia Intensiva desta instituição no ano de 2015 ( $n=79)$. Resultados: Eram homens (58,2\%), idosos $(65,8 \%)$, residentes do DF $(67,1 \%)$, procedentes do Centro Cirúrgico $(69,5 \%)$, o tempo de internação foi de $0-5$ dias $(69,6 \%)$, tinham câncer de cólon e reto $(15,2 \%)$, o tratamento foi cirúrgico $(34,2 \%)$ e morreram (21,5\%). Conclusão: As equipes de Unidades de Terapia Intensiva precisam estar preparadas para assistir ao paciente oncológico em suas individualidades.

Descritores: Perfil de Saúde, Neoplasias, Unidade de Terapia Intensiva.

\begin{abstract}
Objective: This study aimed describing the profile of cancer patients admitted to the adult Intensive Care Unit in the year 2015. Methods: It is a descriptive, retrospective and cross-sectional study, with a quantitative approach, based on the analysis of medical records. This study was developed in a university hospital of the Federal District, in the Medical File Service. The study population consisted of all charts of cancer patients admitted to the Intensive Care Unit of this institution in the year $2015(n=79)$. Results: There were men $(58.2 \%)$, elderly $(65.8 \%)$, residents of the DF $(67.1 \%)$ from the Surgical Center $(69.5 \%)$, hospitalization time was $0-5$ days, (6\%) had colon and rectal cancer (15.2\%), surgical treatment $(34.2 \%)$ and $21.5 \%$ died. Conclusion: Teams off intensive care unit need to be prepared to assist the cancer patient in their individualities.
\end{abstract}

Key words: Health Profile, Neoplasms, Intensive Care Units.

\footnotetext{
1 Universidade de Brasília, Brasília-DF. *E-mail: laurabeatriz.unb@gmail.com

*Este trabalho foi desenvolvido através do Programa de Iniciação Científica da Universidade de Brasília ProlCUnB e a autora correspondente foi Bolsista - Edital 2017-2018.
}

SUBMETIDO EM: 5/2019

ACEITO EM: 6/2019

PUBLICADO EM: $8 / 2019$ 


\section{RESUMEN}

Objetivo: Identificar el perfil sociodemográfico y clínico de los pacientes oncológicos admitidos en una Unidad de Terapia Intensiva Adulto, en el año 2015. Métodos: Se trata de un estudio descriptivo, retrospectivo y transversal, con abordaje cuantitativo, basado en el análisis de prontuarios. El estudio fue desarrollado en un hospital universitario del Distrito Federal, en el Servicio de Archivo Médico. La población del estudio fue compuesta por todos los prontuarios de pacientes oncológicos admitidos en la Unidad de Terapia Intensiva de esta institución en el año $2015(\mathrm{n}=79)$. Resultados: Eran hombres $(58,2 \%)$, ancianos $(65,8 \%)$, residentes del DF (67,1\%), procedentes del Centro Quirúrgico (69,5\%), el tiempo de internación fue de 0-5 días (69,6\%), tenían cáncer de colon y recto $(15,2 \%)$, el tratamiento fue quirúrgico $(34,2 \%)$ y murieron $(21,5 \%)$. Conclusión: Los equipos de Unidades de Terapia Intensiva necesitan estar preparadas para asistir al paciente oncológico en sus individualidades.

Palabras clave: Perfil de Salud, Neoplasias, Unidades de Cuidados Intensivos.

\section{INTRODUÇÃO}

Câncer é considerado um conjunto de mais de 100 doenças distintas e que ainda não se conhece a etiologia. Essas doenças têm em comum o crescimento desordenado de células malignas e formação de tumores malignos, as quais podem invadir tecidos e órgãos adjacentes ou distantes, fenômeno esse chamado de metástase (BRASIL, 2011a), Além disso, o câncer faz parte das Doenças Crônicas Não Transmissíveis (DCNT) que são consideradas uma das principais causas de morte no mundo (BRASIL, 2011b).

Estimou-se para os anos de 2018-2019 a ocorrência de cerca de 600 mil casos novos de câncer, para cada ano no Brasil (incluindo câncer de pele não melanoma). Nessa estimativa destaca-se que os tipos de câncer mais prevalentes no país (exceto o câncer de pele não melanoma) são os de próstata, pulmão, mama feminina e cólon e reto, os quais estão associados aos hábitos de vida urbanizados. Além disso, ainda são altas as taxas de câncer de colo de útero, estômago e esôfago, que são associados a infecções. E ainda, destaca-se que as características sociodemográficas e de saúde das pessoas influenciam no seu perfil oncológico (BRASIL, 2018).

A incorporação de tratamentos inovadores, a expansão tecnológica e a transição epidemiológica, são fatores que têm aumentado à sobrevida e melhorado o prognóstico dos pacientes oncológicos. Assim, cresce também a necessidade de cuidados intensivos daqueles que apresentam possibilidade de cura ou controle do câncer e intercorrências passíveis de reversibilidade (SOARES M, 2008; FAVARIN SS e CAMPONOGARA S, 2012).

A Unidade de Terapia Intensiva (UTI) é definida como uma unidade hospitalar em que acontece atendimento de pacientes graves, realizado por profissionais especializados, de forma contínua, utilizando materiais específicos e tecnologias necessárias ao diagnóstico, monitorização e terapia (BRASIL, 2010).

As últimas décadas foram de grande importância devido aos avanços no diagnóstico precoce e, principalmente, no manuseio de pacientes com câncer. A terapêutica avançada em UTI tem contribuído significativamente para a redução da mortalidade geral por câncer (SOARES M, 2008).

Um estudo comparativo brasileiro realizado em uma UTI de hospital terciário, comparou o desfecho hospitalar de pacientes oncológicos e não oncológicos submetidos a procedimentos cirúrgicos eletivos com alto risco de óbito e mostrou que a mortalidade hospitalar dos pacientes oncológicos não foi superior à dos pacientes sem câncer. (AMENDOLA CP, et al., 2006).

Partindo da perspectiva supracitada, é notável a relevância de um estudo o qual relaciona o paciente oncológico e suas características sociodemográficas e de saúde dentro de uma UTI. Justifica-se ainda, por ser um trabalho único para o local estudado, o que possibilitará conhecer as características sociodemográficas e de saúde desses pacientes de forma a proporcionar condições para um melhor planejamento da assistência em enfermagem e, concomitante a isso, melhora em todo o processo subsequente. 
Sendo assim, este estudo teve como objetivo identificar o perfil sociodemográfico e clínico dos pacientes oncológicos admitidos em uma Unidade de Terapia Intensiva (UTI) adulto de um hospital universitário do Distrito Federal, no ano de 2015 e como objetivos específicos verificar quais tipos de cânceres eram mais prevalentes, identificar o tempo médio de internação e analisar a mortalidade desses pacientes na UTI.

\section{MÉTODO}

Trata-se de um estudo descritivo, retrospectivo e transversal, com abordagem quantitativa, baseado na análise de prontuários.

O estudo foi desenvolvido em um hospital universitário do Distrito Federal, no Serviço de Arquivo Médico (SAME). A UTI em questão tinha 10 leitos ativos para pessoas com idade acima de 15 anos.

A população do estudo foi composta por todos os prontuários de pacientes oncológicos admitidos na Unidade de Terapia Intensiva (UTI) desta instituição.

A seleção dos prontuários foi feita de acordo com o caderno de registro de admissão de pacientes da UTI. Para determinar a amostra, foi considerada a amostragem não probabilística, do tipo amostra por conveniência. Foram analisados 193 prontuários, de pacientes atendidos na UTI no ano de 2015, dos quais 79 (40,9\%) eram de pacientes oncológicos.

Os critérios de inclusão foram: prontuários de pacientes oncológicos de ambos os sexos, admitidos na UTI no período entre primeiro de janeiro de 2015 a 31 de dezembro de 2015. Como critérios de exclusão: prontuários que não estivessem disponíveis no SAME para consulta no dia da coleta.

A coleta de dados foi realizada pelos autores do presente estudo. Havia um questionário auxiliar de pesquisa como fonte de registro desses dados, construído pelos próprios autores com base em informações contidas em publicações sobre o tema.

Foram coletados os seguintes dados: as características sociodemográficas e relacionadas à saúde desses pacientes.

Os dados coletados foram transportados para a planilha de dados Excel for Windows versão 6.0. Foi realizada análise descritiva das variáveis, com uso de tabelas de frequência e medidas de posição (média, mínima e máxima).

O presente estudo atendeu à Resolução CNS 466/2012. O projeto foi aprovado pelo Comitê de Ética da Faculdade de Ciências da Saúde da Universidade de Brasília, sob o parecer no 2.197.661. Foram utilizados apenas e exclusivamente dados secundários dos prontuários de pacientes. Cada prontuário recebeu um número e os dados foram analisados de forma agrupada.

\section{RESULTADOS}

Foram encontrados 193 prontuários, de pacientes atendidos na referida UTI no ano de 2015, dos quais 79 $(40,9 \%)$ eram de pacientes oncológicos. Os dados sociodemográficos destes sujeitos estão apresentados na Tabela 1.

Os pacientes oncológicos eram do sexo masculino (58,2\%), idosos (65,8\%), com média de idade de 62 anos (idade mínima de 22 e máxima de 92 anos).

Em relação à cor da pele, os sujeitos descritos eram pardos $(41,8 \%)$. Em geral eram casados $(51,9 \%)$, provenientes da região nordeste (62\%) e residentes no Distrito Federal $(67,1 \%)$, na Região Administrativa (RA) Ceilândia (12,6\%).

As informações sobre escolaridade dos pacientes não foram encontradas nos prontuários em $72 \%$ dos casos, porém os prontuários que constavam essa informação, a maioria tinha apenas quatro anos de estudo $(10,1 \%)$. As rendas pessoais e familiares não foram encontradas em $100 \%$ dos prontuários analisados 
Tabela 1 - Frequência absoluta e relativa das variáveis sociodemográficas segundo os prontuários dos pacientes oncológicos internados na UTI em 2015. Brasília-DF. 2018. ( $n=79)$.

\begin{tabular}{|c|c|c|}
\hline Variáveis & $\mathbf{n}$ & $\%$ \\
\hline \multicolumn{3}{|l|}{ Sexo } \\
\hline Feminino & 33 & 41,8 \\
\hline Masculino & 46 & 58,2 \\
\hline \multicolumn{3}{|l|}{ Faixa etária } \\
\hline $22-29$ & 03 & 3,8 \\
\hline $30-39$ & 04 & 5,1 \\
\hline $40-49$ & 08 & 10,1 \\
\hline $50-59$ & 12 & 15,2 \\
\hline $60-69$ & 23 & 29,1 \\
\hline $70-79$ & 25 & 31,6 \\
\hline $80-92$ & 04 & 5,1 \\
\hline \multicolumn{3}{|l|}{ Cor da Pele } \\
\hline Branca & 12 & 15,2 \\
\hline Parda & 33 & 41,8 \\
\hline Preta & 06 & 7,6 \\
\hline Informação não encontrada & 28 & 35,4 \\
\hline \multicolumn{3}{|l|}{ Estado civil } \\
\hline Casado & 41 & 51,9 \\
\hline União Estável & 01 & 1,3 \\
\hline Separado/Divorciado & 02 & 2,5 \\
\hline Solteiro & 19 & 24 \\
\hline Viúvo & 10 & 12,7 \\
\hline Informação não encontrada & 06 & 7,6 \\
\hline \multicolumn{3}{|l|}{ Local de residência } \\
\hline Distrito Federal & 53 & 67,1 \\
\hline Goiás & 22 & 27,8 \\
\hline Outros & 04 & 5,1 \\
\hline \multicolumn{3}{|l|}{ Ocupação } \\
\hline Aposentado/Pensionista & 27 & 34,1 \\
\hline Aposentado/Pensionista que trabalham & 04 & 5,1 \\
\hline Trabalhadores & 15 & 19,0 \\
\hline Informação não encontrada & 33 & 41,8 \\
\hline
\end{tabular}

Fonte: Dados da pesquisa, 2019.

Os dados relacionados ao estado geral de saúde dos pacientes analisados, que constavam nos prontuários, estão descritos na Tabela 2. Em geral os pacientes internados na UTI eram provenientes do Centro Cirúrgico $(69,5 \%)$. Cuja maioria ficaram internados de zero a cinco dias $(69,6 \%)$ com tempo médio de 10 dias.

Em apenas 18 prontuários $(22,8 \%)$ foi identificado relato de visita familiar, sendo que, primou a visita de esposas e filhos, consequentemente, em 55 prontuários (69,5\%) essa informação não foi encontrada. Quanto aos principais antecedentes diagnósticos destacam-se, respectivamente, a Hipertensão Arterial Sistêmica $(55,7 \%)$, a Diabetes Mellitus $(22,8 \%)$, as doenças pulmonares $(15,2 \%)$.

Tratando-se da Sepse, desfecho comum em internações de terapia intensiva, a maioria deles não teve descrição de sepse na internação $(50,6 \%)$, porém, em 24 prontuários $(30,4 \%)$ essa informação não foi identificada. Em apenas nove prontuários $(11,4 \%)$ havia relato de reinternação nesta UTI nos últimos meses e as causas majoritárias foram relacionadas à neoplasia $(55,6 \%)$. A maioria dos pacientes apresentou de lesão de pele $(87,3 \%)$, principalmente ferida operatória $(92,8 \%)$.

Referindo-se aos óbitos, 17 (21,5\%) dos sujeitos acometidos por câncer na referida UTI morreram. A principal causa de morte dos pacientes oncológicos foi choque $(58,8 \%)$. Salienta-se que os que não foram a óbito tiveram alta predominantemente para Clínica Cirúrgica (60,7\%). 
Tabela 2 - Frequência absoluta e relativa das variáveis relacionadas à saúde segundo os prontuários dos pacientes oncológicos internados na UTI em 2015. Brasília-DF. 2018.
Variáveis

\section{n}

Procedência antes da UTI

Centro Cirúrgico

55

09

06

03

06

Informação não encontrada

Tempo de Internação na UTI

00-05 dias

09

07

26-120 dias

Principais Antecedentes Diagnósticos*

Hipertensão Arterial Sistêmica

Informação não encontrada

\section{Lesão de Pele}

Ferida operatória

Lesão por pressão

Outros

Informação não encontrada

\section{Hospitalização nos últimos 12 meses}

Sim

Não

Informação não encontrada

\section{Principal Causa de Óbito}

Choque

Neoplasias

Falência de múltiplos órgãos

* UPC: Unidade de Paciente Crítico; CPA: Centro de Pronto-Atendimento; havia mais de um antecedente diagnóstico descrito para o mesmo sujeito.

Fonte: Dados da pesquisa, 2019. 
Os dados acerca dos procedimentos invasivos apresentam-se na Tabela 3. Nos prontuários analisados foi possível identificar que os pacientes internados fizeram uso de Cateter Vesical de Demora (CVD) (91,1\%), Cateter Venoso Central (CVC) (84,4\%), Pressão Arterial Invasiva (PAI) $(69,6 \%)$ e Ventilação Mecânica (VM) $(53,2 \%)$.

O tempo de permanência prevalente desses dispositivos invasivos foi de um a 10 dias, em CVC $(71,6 \%)$, em CVD (69,4\%) e PAI (69,1\%). Quanto ao uso de Drogas Vasoativas (DVA) foi encontrado nos relatos apenas o uso de Noradrenalina.

Tabela 3 - Frequência absoluta e relativa dos procedimentos invasivos realizados durante a internação segundo os prontuários dos pacientes oncológicos internados na UTI em 2015. Brasília-DF. 2018.

\begin{tabular}{|c|c|c|c|c|c|c|c|}
\hline \multirow{2}{*}{$\begin{array}{l}\text { Procedimentos realizados } \\
\text { durante a internação na UTI* }\end{array}$} & \multicolumn{3}{|c|}{ Sim } & \multicolumn{2}{|c|}{ Não } & \multicolumn{2}{|c|}{$\begin{array}{l}\text { Informação não } \\
\text { encontrada }\end{array}$} \\
\hline & & $\mathrm{n}$ & $\%$ & $n$ & $\%$ & $\mathbf{n}$ & $\%$ \\
\hline Hemodiálise & 21 & & 26,6 & 52 & 65,8 & 06 & 7,6 \\
\hline Ventilação Mecânica & 42 & & 53,2 & 31 & 39,2 & 06 & 7,6 \\
\hline Traqueostomia & 16 & & 20,3 & 58 & 73,4 & 05 & 6,3 \\
\hline Dreno de Tórax & 21 & & 26,6 & 49 & 62 & 09 & 11,4 \\
\hline Outros tipos de Drenos & 27 & & 34,2 & 47 & 59,5 & 05 & 6,3 \\
\hline Nutrição Enteral & 34 & & 43,1 & 37 & 46,8 & 08 & 10,1 \\
\hline Cateter Venoso Central & 67 & & 84,8 & 09 & 11,4 & 03 & 3,8 \\
\hline Pressão Arterial Invasiva & 55 & & 69,6 & 18 & 22,8 & 06 & 7,6 \\
\hline Cateter Vesical de Demora & 72 & & 91,1 & 04 & 5,1 & 03 & 3,8 \\
\hline Nutrição Parenteral Total & 05 & & 6,3 & 64 & 81 & 10 & 12,7 \\
\hline Uso de Droga Vasoativa & 21 & & 26,6 & 50 & 63,3 & 08 & 10,1 \\
\hline
\end{tabular}

*Havia mais de um procedimento invasivo descrito para o mesmo sujeito.

Fonte: Dados da pesquisa, 2019.

As informações referentes aos tipos de cânceres desses pacientes estão apresentadas na Tabela 4. Predominou o câncer de cólon e reto (15,2\%), seguidos dos cânceres de bexiga $(8,9 \%)$, esôfago (8,9\%) e pulmão $(8,9 \%)$.

Ao estratificar os dados por sexo, em homens o câncer de cólon e reto foi o mais prevalente $(17,4 \%)$ e em mulheres, nas mamas $(18,2 \%)$.

Em 28 (35,5\%) prontuários foi descrito que o sujeito apresentava metástase, entretanto essa informação não foi encontrada em $39,2 \%$ dos prontuários.

O tratamento para o câncer predominante nos registros foi o cirúrgico, descrito em $34,2 \%$ dos prontuários, seguido da quimioterapia $(12,6 \%)$ e cirurgia mais quimioterapia $(7,6 \%)$.

Também foi observado a associação de três tratamentos (cirúrgico, quimioterapia e radioterapia) para o mesmo indivíduo em $5,1 \%$ dos casos.

Em relação ao estadiamento dos cânceres, não foi possível calcular as proporções devido à falta de informações registradas no prontuário. 
Tabela 4 - Frequência absoluta e relativa das variáveis oncológicas dos prontuários dos pacientes internados na UTI em 2015. Brasília-DF. 2018.

\begin{tabular}{|c|c|c|}
\hline Variáveis & $\mathbf{n}$ & $\%$ \\
\hline \multicolumn{3}{|l|}{ *Tipos de Câncer } \\
\hline Bexiga & 07 & 8,9 \\
\hline Colo de Útero & 03 & 3,8 \\
\hline Cólon/Reto & 12 & 15,2 \\
\hline Endócrino & 04 & 5,1 \\
\hline Esôfago & 07 & 8,9 \\
\hline Estômago & 02 & 2,5 \\
\hline Fígado & 04 & 5,1 \\
\hline Mama & 06 & 7,6 \\
\hline Ovário & 05 & 6,3 \\
\hline Pâncreas & 04 & 5,1 \\
\hline Próstata & 05 & 6,3 \\
\hline Pulmão & 07 & 8,9 \\
\hline $\operatorname{Rim}$ & 05 & 6,3 \\
\hline Outros & 11 & 13,9 \\
\hline \multicolumn{3}{|l|}{ Metástase } \\
\hline Sim & 20 & 25,3 \\
\hline Não & 28 & 35,5 \\
\hline Ignorado & 31 & 39,2 \\
\hline \multicolumn{3}{|l|}{ Cuidado Paliativo } \\
\hline Sim & 06 & 7,6 \\
\hline Não & 48 & 60,8 \\
\hline Ignorado & 25 & 31,6 \\
\hline \multicolumn{3}{|l|}{ Tratamento } \\
\hline Cirúrgico & 27 & 34,2 \\
\hline Quimioterapia & 10 & 12,6 \\
\hline Radioterapia & 02 & 2,5 \\
\hline Cirúrgico + Quimioterapia. & 06 & 7,6 \\
\hline Cirúrgico + Radioterapia. & 01 & 1,3 \\
\hline Cirúrgica + Radioterapia + Quimioterapia & 04 & 5,1 \\
\hline Quimioterapia. + Radioterapia & 03 & 3,8 \\
\hline Ignorado & 26 & 32,9 \\
\hline
\end{tabular}




\section{DISCUSSÃO}

O presente estudo identificou que no ano de 2015, foram internados 193 pacientes na referida UTI, os quais 40,9\% ( $n=79)$ tinham diagnóstico de câncer, resultado consonante com estudos recentes sobre UTIs (FARIAS LL, et al., 2013; EL-FAKHOURI S, et al., 2016; SILVA JB, et al., 2018).

O predomínio do sexo masculino pode estar associado ao fato dos homens serem considerados menos diligentes com a saúde e como consequência, necessitam mais de terapias invasivas e emergenciais (BOS MMEM, et al., 2015; EL-FAKHOURI S, et al., 2016).

Em geral, os idosos têm se destacado em número de internações em UTI (ALMEIDA RMS, et al., 2011; BOS MMEM, et al., 2015; XING X, et al., 2015; EL-FAKHOURI S, et al., 2016). Esse dado é compreendido pela transição demográfica no Brasil e no mundo; uma vez que é notório o aumento da população idosa em detrimento das outras faixas etárias. Esse fenômeno influencia diversos setores da sociedade e com o envelhecimento populacional, há aumento das DCNT, especialmente do câncer (BRASIL, 2011).

Com referência a cor da pele, predominou a cor parda, essa característica foi divergente com a literatura que aponta a cor de pele branca como mais comum em pacientes internados em UTI (EL-FAKHOURI S, et al., 2016). Explica-se esse fato por serem estudos feitos em regiões geográficas distintas.

Os indivíduos eram procedentes da região nordeste e residiam no DF. É sabido por Carvalho ARC (2008) que o fluxo migratório para região do DF é grande desde sua fundação, pois, atraídos pela oportunidade de trabalho, principalmente na construção civil, os nordestinos foram os principais migradores para o planalto central na construção de Brasília. E esse perfil demográfico se mantém até os dias atuais.

Os registros mostraram que $51,9 \%$ dos pacientes oncológicos eram casados, perfil semelhante observado por Melo ACL, et al. (2014). Além disso, também foi semelhante o grau de instrução dos indivíduos, neste trabalho foi identificado apenas quatro anos de estudo para maioria $(10,1 \%)$ dos registros que continham a informação. Infelizmente, essa informação valiosa não foi encontrada em $72 \%$ dos prontuários. Conhecer o grau de instrução dos pacientes permite direcionar a equipe quanto à comunicação efetiva para cada individuo, a qual acarretará em um cuidado mais efetivo e humano. (MELO ACL, et al., 2014)

Não foi encontrada informação sobre a renda pessoal e familiar dos sujeitos nos prontuários analisados, o que impossibilitou conhecer o perfil econômico dessa população. Informações acerca da renda pessoal e familiar também não foram encontradas em estudos análogos (SILVA JB, et al., 2018; EL-FAKHOURI S, et al., 2016; MELO ACL, et al., 2014).

Soares M, et al. (2010) em um estudo multicêntrico, realizado em 28 hospitais diferentes no Brasil, sobre o perfil do paciente oncológico atendido em UTI, evidenciou que o principal motivo de internação desse público nas UTIs foi o cuidado pós-cirúrgico (57\%). Outro trabalho, realizado na China, que analisou a mortalidade dos pacientes com câncer classificou a cirurgia eletiva como principal motivo de internação destes na UTI $(92,9 \%)$ (XING X, et al., 2015). Esses dados corroboram com os encontrados neste estudo.

Em relação ao tempo de internação encontrado, a média foi de 10,4 dias. Tempo similar foi observado na literatura (MELO ACL, et al, 2014; EL-FAKHOURI S, et al., 2016; XING X, et al., 2015).

Ainda sobre a internação, houve relato de visita familiar para $22,8 \%$ dos pacientes, sendo esposa e filhos os mais recorrentes, todavia, $69,5 \%$ dos registros não continham tal descrição. O registro sobre visita e presença de familiares na UTI é imprescindível, pois pode auxiliar no tratamento facilitando a comunicação entre o paciente e a equipe de saúde (BECCARIA LM, et al., 2008).

As principais comorbidades associadas aos pacientes oncológicos foram Hipertensão Arterial Sistêmica $(55,7 \%)$ e Diabetes Mellitus (22,8\%) em concordância com a literatura (FARIAS LL, et al., 2013; SILVA JB, et al., 2018).

Relatos sobre lesão de pele foram encontrados em $87,3 \%$ dos registros, destas, $92,8 \%$ eram feridas operatórias - o que justifica as internações desses pacientes serem provenientes do centro cirúrgico. Além disso, os pacientes que tiveram alta da UTI foram encaminhados em maioria $(60,7 \%)$ para a clínica cirúrgica. 
Estudo brasileiro multicêntrico afirma que "Existem taxas de sobrevivência encorajadoras para pacientes com câncer que necessitam de cuidados intensivos." (SOARES M, et al., 2010).

Em consonância, Lyngaa T, et al. (2015) analisaram o perfil de pacientes que morrem em decorrência de câncer e doenças crônicas não cancerígenas internadas em UTIs, nesta pesquisa 35,5\% dos pacientes com doenças não cancerígenas morreram, por outro lado, 29,2\% dos pacientes com câncer foram a óbito; sendo assim, foi identificado risco aumentando de morrer por doenças não cancerígenas nas UTIs estudadas, o que também foi verificado nos achados deste estudo.

Procedimentos invasivos são comumente utilizados em UTIs para manutenção da vida dos pacientes, dentre estes estão os cateteres vesicais, acessos vasculares e ventilação mecânica (SOUSA MAS, et al., 2017).

De consonante modo, foi identificado uso de CVD em 91,1\%, CVC em 84,8\%, PAI em 69,6\% e VM em $53,2 \%$ dos pacientes.

Sousa MAS, et al. (2017) alerta para a relação dos procedimentos citados com as Infecções Relacionadas com a Assistência à Saúde (IRAS), sugerindo que o controle do tempo de permanência é um modo razoável de prevenção das IRAS. Nesta perspectiva, o presente estudo verificou tempo de permanência de 1-10 dias como mais frequente dos procedimentos CVC $(71,6 \%)$, CVD $(69,4 \%)$ e PAI $(69,1)$.

O câncer de cólon e reto é terceiro tipo mais prevalente e o quarto que mais mata no mundo. Está relacionado a muitos fatores, dentre eles, os genéticos, as complicações infecciosas e principalmente os hábitos de vida ocidentalizados: consumo de alimentos industrializados, inatividade física, consumo de álcool e tabaco, entre outros. Portanto, em países desenvolvidos e em desenvolvimento este tipo de neoplasia maligna é mais prevalente (BRASIL, 2018).

No presente estudo o câncer de cólon e reto foi o mais comum 15,2\%; ao segmentar-se por sexo, em homens se manteve cólon e reto $(17,4 \%)$, mas entre as mulheres, o mais comum foi o câncer de mamas $(18,2 \%)$, dados similares aos que Xing X, et al. (2015) encontraram.

O tratamento cirúrgico predominou (34,2\%), contudo, também se observou a presença de associação de tratamentos como cirurgia e quimioterapia $(7,6 \%)$, e cirurgia, quimioterapia e radioterapia $(5,1 \%)$; dados semelhantes foram encontrados em estudo internacionais sobre o paciente oncológico na UTI (BOS MMEM, et al., 2015; XING X, et al., 2015, SOARES M, et al. 2010).

Bos MMEM et al. (2015) também aponta que a associação de tratamentos aumenta o risco de o paciente oncológico precisar de cuidados intensivos, haja vista que são tratamentos agressivos e que causam efeitos adversos relacionados a imunidade, sendo a neutropenia a mais comum.

Nesta pesquisa, as principais limitações encontradas foram: a falta de informações importantes nos registros, a desorganização cronológica dos anexos e dados de pacientes diferentes contidos no mesmo prontuário. Sugere-se o incentivo a melhores anotações e organização dos dados nos prontuários, para que possibilite mais pesquisas na área além de dados consistentes.

\section{CONCLUSÃO}

Conclui-se que 40,9\% dos pacientes admitidos na UTI tinham diagnóstico de câncer. Eram homens, idosos, com idade média de 62 anos, casados, pardos, procedentes da região nordeste e residentes do DF. Foram encaminhados para UTI do centro cirúrgico, o tempo de internação foi de 0-5 dias, tinham lesão de pele em que se destacou a ferida operatória. Aqueles que tiveram alta foram para clínica cirúrgica. $O$ câncer mais prevalente foi o câncer de cólon e reto e o tratamento foi cirúrgico.

Portanto, para uma assistência de qualidade é necessário o conhecimento do perfil sociodemográfico e clínico dos sujeitos, haja vista que desse modo, a equipe de saúde poderá traçar planos de cuidados mais efetivos e condizentes com a população assistida. 


\section{REFERÊNCIAS 985}

1. ALMEIDA RMS de, et al. Perfil dos pacientes de pós-operatório oncológico em centro de terapia intensiva. Rev. Med. Minas Gerais, 2011; 21(2): 145-151.

2. AMENDOLA CP, et al. A Doença Oncológica não deve ser um Fator Limitante para Admissão na UTI de Pacientes Submetidos a Cirurgias de Alto Risco. Revista Brasileira de Terapia Intensiva, 2006. v. 18, n. 3, p. $251-255$.

3. BECCARIA LM, RIBEIRO R, SOUZA GL, et al. Visita em Unidades de Terapia Intensiva: concepção dos familiares quanto à humanização do atendimento. Arq. Ciênc. Saúde, 2008. v. 15, n. 2, p.65-69, abr./jun..

4. BOS MMEM, VERBURG IWM, DUMAIJ I et al. Intensive care admission of cancer patients: a comparative analysis. Cancer Medicine. 2015, 4(7):966-976.

5. BRASIL. Ministério da Saúde. Instituto Nacional do Câncer José de Alencar Gomes da Silva. INCA - Instituto Nacional de Câncer - ABC do câncer: abordagens básicas para o controle do câncer. 2011, Rio Janeiro.

6. BRASIL. Ministério da Saúde. Plano de ações estratégicas para o enfrentamento de Doenças Crônicas Não Transmissíveis (DCNT) no Brasil 2011-2022. 2011, Brasília-DF. B

7. BRASIL. Ministério da Saúde. Instituto Nacional do Câncer José de Alencar Gomes da Silva. INCA - Instituto Nacional de Câncer - Estimativa 2018-2019. 2018, Rio Janeiro.

8. BRASIL. Agência Nacional de Vigilância Sanitária (ANVISA). Resolução N 7, de 24 de fevereiro de 2010. Dispõe sobre os requisitos mínimos para funcionamento de Unidades de Terapia Intensiva e dá outras providências. 2010, fev.

9. CARVALHO ARC. Migrantes em Brasília: os motivos, as dores e os sonhos numa perspectiva clínica. $97 f$. Dissertação de Mestrado. 2008. Universidade de Brasília, Brasília.

10. EL-FAKHOURI S, et al. Epidemiological profile of ICU patients at Faculdade de Medicina de Marília. Rev. Assoc. Med. Bras. 2016, 62(3):248-254.

11. FARIAS LL, et al. Perfil clínico e laboratorial de pacientes com sepse, sepse grave e choque séptico admitidos em uma unidade de terapia intensiva. Rev. Saúde Públ. Santa Cat. Florianópolis, 2013. v. 6, n. 3, p. 50-60, jul./set.

12. FAVARIN SS e CAMPONOGARA S. Perfil Dos Pacientes Internados Na Unidade De Terapia Intensiva Adulto De Um Hospital Universitário. Revista Enfermagem UFSM. 2012. v. 2, n. 2, p. 320-329.

13. LYNGAA T, et al. Intensive care at the end of life in patients dying due to non-cancer chronic diseases versus cancer: a nationwide study in Denmark. Critical Care. 2015,19:413.

14. MELO ACL, et al. Perfil de pacientes de terapia intensiva: subsídios para a equipe de enfermagem. Rev. Enferm. UFPE online. Recife, 2014. 8(9):3142-8.

15. SILVA JB, et al. Perfil clínico de longevos em uma unidade de terapia intensiva. Acta Paul Enferm. 2018. 31(1):3945, .

16. SOARES M. Quando o tratamento oncológico pode ser fútil? Do ponto de vista do intensivista. Revista Brasileira de Cancerologia. 2008.54(4): 389-392.

17. SOARES M, CARUSO P, TELES JM. et al. Characteristics and outcomes of patients with cancer requiring admission to intensive care units: a prospective multicenter study. Crit. Care Med. 2010. 38(1):9-15.

18. SOUSA MAS, et al. Infecções hospitalares relacionadas a procedimentos invasivos em unidades de terapia intensiva: revisão integrativa. Rev Pre Infec e Saúde. 2017. 3(3):49-58.

19. XING X, et al. Performance of Three Prognostic Models in Patients with Cancer in Need of Intensive Care in a Medical Center in China. PLoS. 2015. ONE 10(6): e0131329. 